FERMULAB-Conf-91/258

(E-731)

\title{
The Neutral Kaon Program at Fermilab and Recent E731 Results
}

\author{
E. Ramberg \\ Fermi National Accelerator Laboratory \\ P.O. Box 500, Batavia, Illinois 60510
}

September 1991

* Presented at the 3rd Topical Seminar on Heavy Flavors, San Miniato, Italy, June 1991. 



\section{THE NEUTRAL KAON PROGRAM AT FERMILAB AND RECENT E731 RESULTS}

\section{Erik J. RAMBERG}

Fermi National Accelerator Laboratory, Batavia, Illinois, 60510

Currently, Fermilab has a long-range program in neutral kaon physics which covers a wide variety of physics topics. In this report, I will be discussing the latest results from experiment E731 on the radiative decay of neutral kaons $K_{L, S} \rightarrow \pi^{+} \pi^{-} \gamma$. These results include the first measurement of two new CP violation parameters: $\eta_{+-\gamma}=(2.60 \pm 0.54 \pm 0.21) \cdot 10^{-3}$, and $\phi_{+-\gamma}=(41 \pm 28 \pm 11)^{\circ}$. The presence of a rho propogator form factor in the $K_{L}$ direct emission decay has been confirmed. Besides these results, the status of experiments E773 and E799, which are running in the 1991 fixed target run at Fermilab, and plans for future neutral kaon experiments will be discussed.

\section{THE FERMILAB NEUTRAL KAON BEAMLINE}

\subsection{Beam Production}

Experiments E773 and E799 are currently running in the same fixed target beamline that was used for experiment E731 in 1987-1988. It is located in the central part of the meson area at Fermilab and delivers a beam composed mainly of neutrons, $K_{L}$ mesons and lambdas. A primary beam of $800 \mathrm{GeV}$ protons impinges on a beryllium target, after which the protons are absorbed. At a horizontal angle of 5 milliradians a beam-defining system of collimators, absorbers and sweeping magnets produces two parallel beams of neutral particles separated in the vertical direction. The width of these beams is approximately $4 \mathrm{~cm}$ square when they enter the experimental apparatus which begins $100 \mathrm{~m}$ downstream of the target. The energy of the $K_{L}$ 's detected in our apparatus averages approximately $70 \mathrm{GeV}$.

\subsection{The E731 Detector}

The E731 apparatus is shown schematically in Figure 1. The goal of the experiment was to measure the direct CP violating parameter $\frac{\dot{s}}{\varepsilon}$ and concentrated on high acceptance and accurate measurement of $t$ wo pion decays, both neutral and charged. ${ }^{1}$ It consisted of a vacuum decay vessel followed by four sets of drift chambers with better than 120 micron resolution, two sets on either side of an analyzing magnet. Following the drift chambers was an array of lead glass crystals for photon detection, with an energy resolution for photons of $2.5 \%+5 \% / \sqrt{E}$. Photon vetoing counters existed at the entrance of the experimental hall, at 4 stations inside the vacuum vessel and in front of the lead glass array. These counters were used to define the aperture for kaon decays. A very thin set of scintillating counters existed about halfway down the vacuum vessel and were used to trigger for charged decays. ${ }^{2}$

One of the main features of the experiment was a 2 interaction length $K_{S}$ regenerator, made of $B_{4} C$, situated at the beginning of the vacuum decay vessel. It alternated in position between the two neutral beams approximately every minute. This regenerator produced $K_{S}$ 's through the well understood mechanism of $K_{L}-K_{S}$ oscillations in matter, and the regenerated $K_{S}$ were subsequently observed to decay in the apparatus. The other neutral beam was not altered in any way and this "vac- 


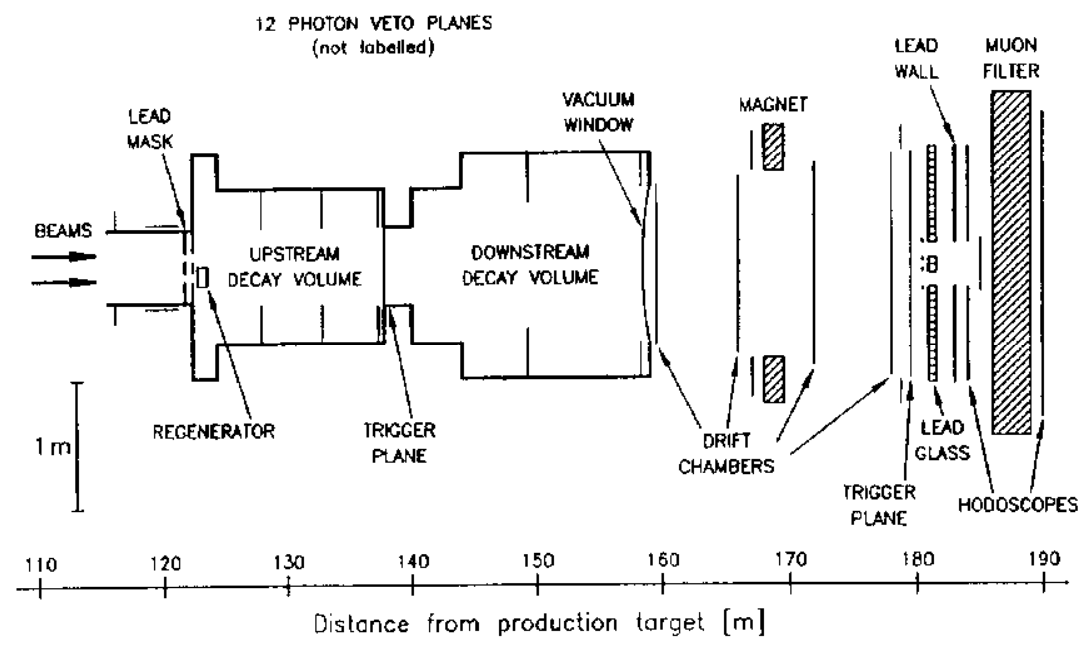

Figure 1: Plan view of the E731 apparatus.

uum beam" contained mostly $K_{L}$ decays. Both $K_{L}$ and $K_{S}$ decays could then be observed during the same running conditions, thus decreasing the systematic error in any comparison between the two types of decays.

\section{E731 RESULTS FOR $K^{\circ} \rightarrow \pi^{+} \pi^{-} \gamma$}

\subsection{Introduction}

In the invariant mass plot of the two charged track events in the E731 data, a background tail is evident at a value lower than the nominal $K^{\circ}$ mass (see Figure 2). Much of this background is due to the $\pi^{+} \pi^{-}$decay with a radiative photon. This section discusses the physics of this decay and our analysis of these events, including the largest sample to date of $K_{L}$ decays into $\pi^{+} \pi^{-} \gamma$.

2.2 Physics of the Decay

The $\pi^{+} \pi^{-} \gamma$ decay for both $K_{L}^{\circ}$ and $K_{S}^{\circ}$ can occur through an inner bremsstrahlung process, termed " $I B$ ", where the photon is emitted by one of the final state pions through an E1 transition. Because this process is similar to the $\pi^{+} \pi^{-}$decay mode, the CP characteristics should be similar, nameiy CP conserving for $K_{S}$ and CP violating for $K_{L}$. However, another type of decay can occur. The direct emission, or "DE" decay, has a photon arising directly from the decay vertex, through an M1 transition. This type of decay should be CP conserving for $K_{L}$ and CP violating for $K_{S} \cdot{ }^{3}$ At present, the DE decay has not been seen in $K_{S}$ decays, while it is clearly evident above the IB background for $K_{L}{ }^{4,5}$ There have been several recent theoretical analyses of the $K_{L} \mathrm{DE}$ decay. ${ }^{8-8}$ Presently, they disagree on whether the amplitude for this decay contains a significant dependence on the photon energy, beyond the standard M1 amplitude. Specifically, it has been postulated that a rho propogator form factor will be present for this decay. ${ }^{6}$ The CP violation parameters for the $\pi^{+} \pi^{-} \gamma$ decay should be the same as for the $\pi^{+} \pi^{-}$decay, once the presence of the DE decay has been taken into account. Although the amount of direct CP-violation is expected to be small, its presence would show up in the DE process by interference with the IB decay.

\subsection{Data Selection}

The trigger for charged decays, of which the $\pi^{+} \pi^{-} \gamma$ 




Figure 2: $M_{\pi+\pi}$ for charged decays from E731. Arrow indicates presence of $\pi^{+} \pi^{2} \gamma$ decays.

decays are a subset, demanded that two charged tracks traversed the detector on either side of the vertical midplane, as determined by the second set of drift chambers and a hodoscope array before the lead glass. No requirement was made on the presence or absence of energy in the lead-glass array. Each event was required in the offline analysis to contain two reconstructed tracks of at least $7 \mathrm{GeV} / \mathrm{c}$ in momentum and have exactly one cluster of energy of at least $1.5 \mathrm{GeV}$ in the lead glass calorimeter not associated with either track. The photon energy in the center of mass system had to be at least $20 \mathrm{MeV}$. To exclude electrons from the sample, we required $\frac{E}{p}<0.8$, where $\mathrm{E}$ is the cluster energy associated with a track and $p$ is the momentum of that track. Each event was also required to pass the same cuts as for analyzing the $\pi^{+} \pi^{-}$ events, which included a total kaon energy between 30 and $160 \mathrm{GeV}$, a reconstructed mass between 484 and $512 \mathrm{MeV} / \mathrm{c}^{2}$ and a total transverse momentum squared of less than $250(\mathrm{MeV} / \mathrm{c})^{2}$. One last cut was made using the variable

$p_{\pi^{\circ}}^{2}=\frac{\left[\left(M_{K^{\circ}}^{2}-M_{\pi^{\mathrm{a}}}^{2}-M_{c}^{2}\right)^{2}-4 M_{\pi^{\circ}}^{2} M_{c}^{2}-4 M_{K^{\circ}}^{2}\left(p_{T}\right)_{c}^{2}\right]}{\left(p_{T}\right)_{c}^{2}+M_{c}^{2}}$

where $M_{c}$ is the invariant mass of the two pions and $\left(p_{T}\right)_{c}$ is their combined transverse momentum. This variable is positive (barring fluctuations in the experimental measurement) if the decay is a $\pi^{+} \pi^{-} \pi^{\circ}$ decay of the kaon, with one photon unobserved. Our cut of $P_{\pi^{\circ}}^{2}<-.05$ reduces the background from the $\pi^{+} \pi^{-} \pi^{\circ}$ decay by approximately a factor of 80 , while retaining $86 \%$ of the $\pi^{+} \pi^{-} \gamma$ decays, as determined from Monte Carlo simulation.

2.4 Branching Ratios and Photon Spectrum

Only a small amount of background remains after the selection cuts, mostly associated with the vacuum beam. There are 9031 decays that pass all of the cuts, 4860 of which are associated with the regenerator beam and 4171 from the vacuum beam. The background under the mass peak was estimated to be $137 \pm 10$ for the vacuum beam and $50 \pm 20$ for the regenerator bearn by studying the $p_{T}^{2}$ distribution of events on either side of the mass peak. The data is shown in Figure 3 along with an estimate for the background level.

A background for the regenerator beam which passes the event cuts is due to the $K_{L} \mathrm{DE}$ component which decays downstrearn of the regenerator. Scaling the number of DE vacuum beam decays after the regenerator position by an absorption factor of 15.7 gives an estimate for this additional number of background events in the regenerator beam to be $96 \pm 4$. The absorption factor is due to absorption of the $K_{L}$ beam as it passes through the regenerator and a moveable absorber that was upstream of the regenerator and followed its motion. These estimates for the number of background events were subtracted from the data sample.

A final correction was made to the data because the 



Figure 3: a) Mass spectrum of vacuum beam events passing all cuts except for the mass cut. Shown as background is the same distribution with the cut $250<p_{T}^{2}<500(\mathrm{MeV} / \mathrm{c})^{2}$ b) Mass spectrum and background of regenerator beam events. c) $p_{T}^{2}$ spectrum of vacuum beam events passing all cuts except for the $p_{T}^{2}$ cut. The background estimate comes from the sidebands on either side of the mass region $.484<M_{\pi \pi \gamma}<.512\left(\mathrm{GeV} / \mathrm{c}^{2}\right)$. d) $p_{T}^{2}$ spectrum and background for the regenerator beam events. (Arrows show cut values.)

requirement of having exactly one unmatched cluster in the calorimeter excludes some events, i.e. those events with accidental activity in the lead glass or with particularly large hadronic showers. By analysing the data with no requirement on the number of extra clusters and selecting those events that passed the mass and transverse momentum cuts with any one of the extra clusters, it was determined that the fraction of events that had exactiy one cluster was $.974 \pm .020$ for $K_{L}$ and $.960 \pm .020$ for $K_{S}$. The number of events of each type were corrected for this effect.

To isolate the separate components of the $K_{L}$ decay into DE and IB, the shape of the photon energy spectrum was used. Figure 4 shows the center of mass photon energy spectrum for the $K_{L}$ decays with the $K_{S}$ decay spectrum superimposed. The $K_{S}$ spectrum has been normalized to fit the $K_{L}$ spectrum in this figure where the normalization was obtained by fitting the $K_{L}$ data with a linear combination of the $K_{S}$ spectrum and a pure DE spectrum obtained from a Monte Carlo simulation containing a rho propogator correction. The normalized $K_{S}$ spectrum can then be associated with the IB component of the $K_{L}$ decays. When subtracted from the total spectrum the DE component for $K_{L}$ decays is then determined.

After determining the numbers of each type of decay, we corrected these numbers according to their acceptance, as determined from an identical analysis of Monte 


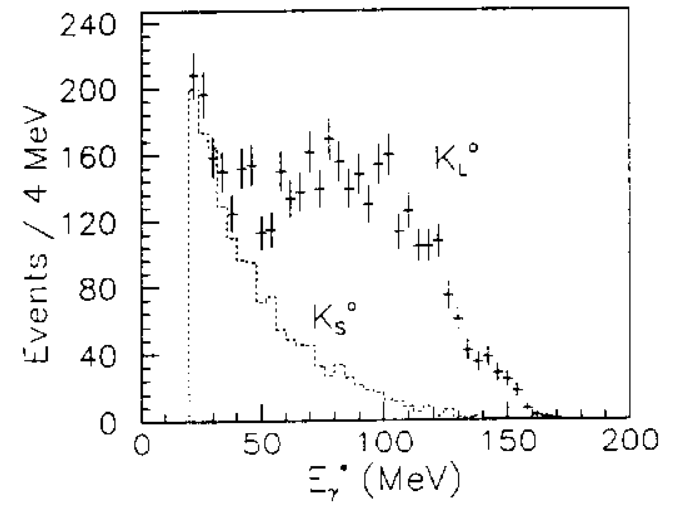

Figure 4: The data points indicate the $E_{\gamma}^{*}$ spectrum for the $K_{L}$ data. The dotted line is the same spectrum for the $K_{S}$ data, normalized as indicated in the text.

Carlo simulated events. Because the decay into $\pi^{+} \pi^{-}$ is statistically a large data sample (over 300,000 events for $K_{L}$ decays and 1.2 million events for $K_{S}$ decays), we normalized the number of $\pi^{+} \pi^{-} \gamma$ decays to that sample, corrected for its own acceptance.

The final results for the numbers of events (after all seiection cuts, background subtractions and corrections) and branching ratios of neutral kaons into $\pi^{+} \pi^{-} \gamma$ are given in Table 1. Also shown, for comparison to a previous result, is the branching ratio for $K_{S}$ decays where $E_{\gamma}^{*}>50 \mathrm{MeV}$. All errors quoted are combined statistical and systematic. Systematic errors include the errors in background subtraction and extra cluster correction quoted above, an error of $1.0 \%$ in the normalization of the $\pi^{+} \pi^{-}$decays, and an estimate for an error in acceptance correction of approximately $1.0 \%$ obtained from the maximum variance seen in the acceptance of Monte Carlo data after varying the cut values in the analysis, including the energy cut for the photon, the cut on the decay vertex position, the minimum track energy, the transverse momentum cut and the cut on the variable $P_{\pi^{\circ}}^{2}$.

A comparison of the shape of the $K_{L} \mathrm{DE}$ photon energy spectrum to the one predicted by two different models for the decay is shown in Figure 5 . As can be clearly seen, the data supports a modification to the standard M1 amplitude that includes a $\rho$ propogator form factor. The $\chi^{2} / D . O . F$. for the fit of the Monte Carlo curve to the data is 1.3 with the energy dependent $\rho$ propagator form factor and 2.5 without it.

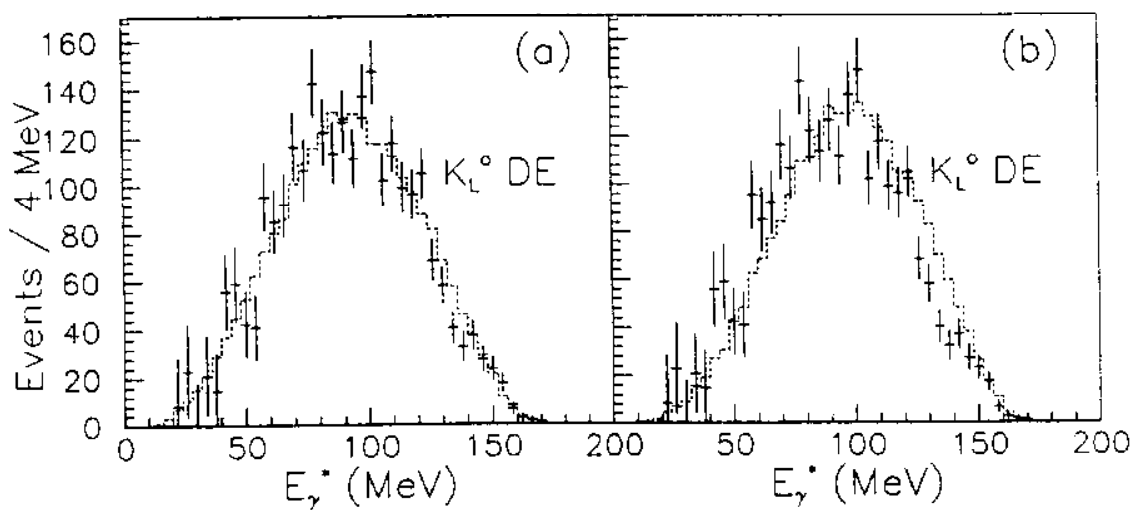

Figure 5: a) The DE $E_{\gamma}^{*}$ spectrum for $K_{L}$ decays compared to the prediction with intermediate vector states. b) Same spectrum compared to the prediction without those states. Fitting to the predicted curves gives a $\chi^{2} /$ D.O.F. of 1.3 for a) and 2.5 for $b$ ). 
Table 1: Branching Ratios for $K_{L, S} \rightarrow \pi^{+} \pi^{-} \gamma$. All errors are combined statistical and systematic. $E_{\gamma}^{*}>20$ $\mathrm{MeV}$ except where noted.

\begin{tabular}{lccc} 
Decay Mode & No. of Evts. & $\Gamma\left(K \rightarrow \pi^{+} \pi^{-} \gamma\right) / \Gamma\left(K \rightarrow \pi^{+} \pi^{-}\right)$ & B.R. \\
\hline $\bar{K}_{L}$ & $4034 \pm 66$ & $(21.9 \pm 0.7) \cdot 10^{-3}$ & $(4.44 \pm .17) \cdot 10^{-6}$ \\
$K_{L}-$ DE only & $2490 \pm 70$ & $(15.0 \pm 0.6) \cdot 10^{-3}$ & $(3.04 \pm .14) \cdot 10^{-5}$ \\
$K_{L}-$ IB only & $1544 \pm 24$ & $(6.90 \pm 0.21) \cdot 10^{-3}$ & $(1.40 \pm .05) \cdot 10^{-5}$ \\
$K_{S}$ & $4714 \pm 73$ & $(6.69 \pm 0.20) \cdot 10^{-3}$ & $(4.59 \pm .14) \cdot 10^{-3}$ \\
$K_{S}-\left(E_{\gamma}^{*}>50 \mathrm{MeV}\right)$ & $1662 \pm 43$ & $(2.49 \pm 0.10) \cdot 10^{-3}$ & $(1.71 \pm .07) \cdot 10^{-3}$
\end{tabular}

\subsection{Determination of $\eta_{+-\gamma}$}

Downstream of the regenerator, the number of decays into the CP-even IB final state can be described as a function of proper time, $\tau$, as

$$
\begin{array}{cc}
\frac{d N}{d \tau} \propto & |\rho|^{2} e^{-\Gamma_{S} \tau}+\left|\eta_{+-\gamma}\right|^{2} e^{-\Gamma_{L} \tau}+ \\
& 2|\rho| \cdot\left|\eta_{+-\gamma}\right| \cos \left(\Delta m \tau+\phi_{\rho}-\phi_{\eta} e^{-\frac{\left(\Gamma_{S}+\Gamma_{L}\right)}{2} \tau}\right)
\end{array}
$$

where $\Gamma_{L}$ and $\Gamma_{S}$ are the decay widths of the $K_{S}^{\circ}$ and $K_{L}^{\circ}$ and $\Delta m$ is their mass difference. The parameter $\rho$ is the regeneration amplitude and is dependent on the kaon momentum, $p_{K}$ (in $\mathrm{GeV}$ ):

$$
|\rho| \propto g p_{K}^{-\alpha}
$$

where $g$ is a factor depending on the length and density of the regenerator. (From an extensive analysis of the $2 \pi$ decay modes, it has been determined that $\alpha=.60$ for our regenerator.) The phase of $\rho$ is then predicted once $\alpha$ and $g$ are known.

The variable $\eta_{+-\gamma}$ in Equation 1 is the CP violating amplitude for the $\pi^{+} \pi^{-} \gamma$ decay mode and is defined as the ratio

$$
\frac{A\left(K_{L} \rightarrow \pi^{+} \pi^{-} \gamma\right)}{A\left(K_{S} \rightarrow \pi^{+} \pi^{-} \gamma\right)}
$$

Previous to the measurement discussed here, only two decay modes have had this amplitude measured, the $\pi^{+} \pi^{-}$ and $\pi^{\circ} \pi^{\circ}$ modes, with: ${ }^{\circ}$

$$
\begin{gathered}
\left|\eta_{+-}\right|=(2.268 \pm 0.023) \cdot 10^{-3} \\
\phi_{+-}=(46.0 \pm 1.2)^{\circ} \\
\left|\eta_{00}\right|=(2.253 \pm 0.024) \cdot 10^{-3} \\
\phi_{00}=(48.5 \pm 3.1)^{\circ}
\end{gathered}
$$

If these amplitudes differ from the value expected due to $K_{L}-K_{S}$ mixing by a small amount, this difference could be attributed to "direct CP violation", or CP violation arising in the decay of the kaons, thought to exist due to Standard Model effects.

The determination of these parameters for the decay $\pi^{+} \pi^{-} \gamma$ is possible using the data obtained after the selection cuts described in the previous section. Because the amount of direct CP violation is expected to be small, the values for $\eta_{+-\gamma}$ and $\phi_{+-\gamma}$ should be similar to the values for the two pion decay modes.

The presence of the DE type of decay can possibly complicate the analysis, however. If no mixing occurs between the DE and IB forms of decay, then the DE decay will modify Equation 1 by adding a term

$$
\frac{f}{(1-f)}\left|\eta_{+-\gamma}\right|^{2} e^{-\Gamma_{L} \tau}
$$

where $\mathrm{f}$ is the fraction of $K_{L}^{\circ}$ decays that occur through the DE process. If, however, mixing does occur between the two forms of decay (meaning E1 transitions occur for the DE process), then the interference term of Equation 
1 will also be affected, thus forcing the fit value of $\eta_{+-\gamma}$ to differ from $\eta_{+-}$. This would indicate that direct CP violation exists in this decay mode, with $\epsilon_{+-\gamma}^{\prime} \neq 0$.

Figure 6a shows the proper time distribution from the regenerator of our data sample. Superimposed on this figure is the same distribution for Monte Carlo simulated $\pi \pi \gamma$ decays in our apparatus, normalized to the same number of events. The value for $\eta_{+-\gamma}$ in the simulation was taken to be the same as $\eta_{+-}$. Shown in Figure $6 \mathrm{~b}$ is the same plot, except that in this instance the Monte Carlo events were generated with no interference term between $K_{L}^{\circ}$ and $K_{S}^{\circ}$ decays. As this figure shows, the data requires the existence of such an interference term, demonstrating for the first time that $K_{L}-K_{S}$ mixing occurs in this decay.

The regenerator beam data sample described above was summed into bins of momentum and $z$ vertex with bin widths of $10 \mathrm{GeV}$ and 2 meters, respectively. An acceptance correction was applied to each bin as determined from a Monte Carlo event sample with at least 10 times the statistics as the data. (The simulation used the amplitude for DE decay containing a $\rho$ propogator term as discussed in the previous section.)

A fit was then performed using a modified form of equation 1 with the $\mathrm{DE}$ addition as shown in equation 5 and with the variable $\tau$ replaced by the seperate variables of momentum and $z$ vertex. The variables allowed to float in the fit were $\eta_{+-\gamma}$ and $\phi_{+-\gamma}$ and $\alpha$, the power law for the regenerator. No direct $\mathrm{CP}$ violation arising from the DE decay was allowed in the fit, so that if such an effect exists, it will modify the fit value of $\eta_{+-\gamma}$.

The result of the fit is

$$
\begin{gathered}
\left|\eta_{+-\gamma}\right|=(2.60 \pm 0.54 \pm 0.21) \cdot 10^{-3} \\
\phi_{+-\gamma}=(41 \pm 28 \pm 11)^{\circ}
\end{gathered}
$$
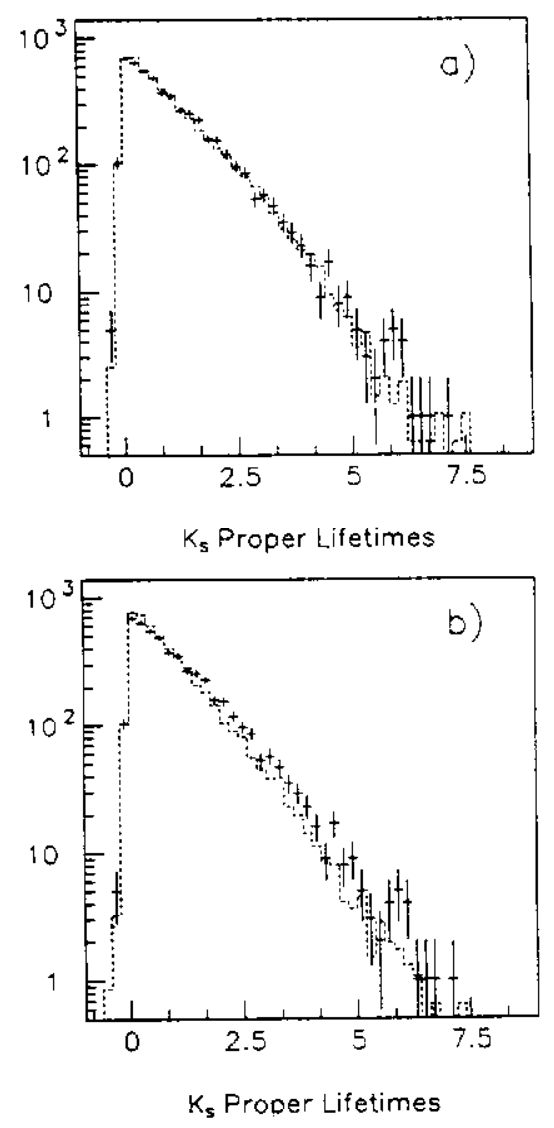

Figure 6: The proper time distribution of $\pi^{+} \pi^{-} \gamma$ decays with respect to the regenerator. Figure a) includes a comparison with Monte Carlo events containing an interference term between $K_{S}$ and $K_{L}$ while figure b) comparison is with simulated events having no such interference term.

The first error is from the statistical uncertainty of the fit as determined by the fitting program while the second error is an estimate of the systematic error. م

The fit value for $\alpha$ was $.68 \pm .04$, which agrees within statistical errors to the value of .60 determined from other decay modes. The $\chi^{2}$ of the fit is 56 for 59 degrees of freedom. 
These results imply that the level of direct CP violation in this decay is small. If all of the difference between the fit value of $\eta_{+-\gamma}$ and the known value of $\eta_{+-}$were to be attributed to a direct $\mathrm{CP}$ violation effect in the $\mathrm{DE}$ decay, and if the phase angles of these two parameters are assumed to be the same, then a limit of

$$
\left|\epsilon_{+-\gamma}^{\prime}\right|<1.2 \cdot 10^{-3}
$$

can be placed at the $95 \%$ confidence level.

\section{CURRENTLY RUNNING EXPERIMENTS}

Currently, the neutral kaon program at Fermilab is continuing with two seperate experiments being performed in the 1991 fixed target run.

\subsection{Experiment E773 - Determination of $\Delta \phi$}

The first $K^{\circ}$ experiment to be performed this year is E773. The goal of this experiment is to measure the phase difference between the two CP violation parameters $\eta_{+-}$and $\eta_{00}$ to a statistical accuracy of about $1 / 3$ of a degree. This phase difference, defined as $\Delta \phi=\phi_{+-}-\phi_{00}$, is predicted to be quite close to zero if CPT is a valid symmetry. Currently, the best measurement of this variable is $\Delta \phi=-0.6 \pm 1.6$, obtained from an analysis of E731 data.

To accomplish this goal, the twin $K_{L}$ beamline structure was maintained and an additional regenerator was placed downstream of the position of the single regenerator in E731. Thus each beam strikes a regenerator. Each regenerator alternates between the two beams every minute. Both regenerators are actively read out with phototubes so that the effect of incoherent scatters inside the material could be studied. The regenerators are matched in length such that the rate of decays in both beams is approximately equal, downstream of the farthest regenerator. The length of the upstream regenerator and the relative position of the two are such that the $K_{L}-K_{S}$ interference term in the decay amplitude is maximized in the downstream region. To accomodate the new regenerator, a section of the vacuum vessel in the region near the $\mathrm{z}$ position of $128 \mathrm{~m}$ was taken out. The upstream regenerator was placed at approximately $z=118$ meters from the target. The trigger scintillators inside the vacuum vessel were removed so that our accesible decay length has been increased by about a factor of two. Between the two regenenerators two new veto counters were placed inside the vacuum decay vessel. Besides these additional detectors, an entirely new triggering scheme was implemented and a new data acquisition system was used, resulting in a data-taking rate of about 14,000 events to tape during each 23 second spill.

\subsection{Experiment E799 - A Rare Decay Search}

The purpose of experiment E799 is to search for the rare decay mode $K_{L} \rightarrow \pi^{\circ} e^{+} e^{-}$, whose amplitude is thought to contain a large component of direct $C P$ violation. Besides this decay mode, the experiment will be sensitive to many others. The sensitivity of experiment E799 to various rare decay modes is shown in Table 2, along with the expected number of events we will see.

Table 2: Expected numbers of events in E799 Phase I compared to best to date for different decay modes of the $K_{L}$. Numbers in parantheses are single event senaitivities when no events are expected.

\begin{tabular}{lcc} 
Decay Mode & \# Events & Best to date \\
\hline$K_{L} \rightarrow \pi^{\circ} e^{+} e^{-}$ & $0\left(<10^{-10}\right)$ & $0\left(<1.8 \cdot 10^{-8}\right)$ \\
$K_{L} \rightarrow \pi^{\circ} \mu^{+} \mu^{-}$ & $0\left(<2 \cdot 10^{-10}\right)$ & $0\left(<1.2 \cdot 10^{-8}\right)$ \\
$K_{L} \rightarrow e^{+} e^{-} e^{+} e^{-}$ & $10-20$ & $0\left(<2.6 \cdot 10^{-8}\right)$ \\
$K_{L} \rightarrow \pi^{+} \pi^{-} e^{+} e^{-}$ & $25-50$ & $0\left(<2.5 \cdot 10^{-6}\right)$ \\
$K_{L} \rightarrow \mu^{+} \mu^{-} \gamma$ & $100-200$ & 2 \\
$K_{L} \rightarrow \pi^{\circ} \gamma \gamma$ & $1000-2000$ & 75
\end{tabular}


The experiment will run in two stages. The first phase is running in 1991 while the second phase is planned to occur in conjunction with a new measurement of $\epsilon^{\prime} / \epsilon$ in the next fixed-target run at Fermilab in 1994.

Many modifications are being made to the apparatus. No regenerators are present in either beam and the vacuum vessel is thus essentially continuous all the way from the target to the first drift chamber, a total distance of 160 meters. Many additional detectors are introduced into the E799 setup besides the veto counters which were included for E773. Specifically, there are 3 planes of transition radiation detectors (TRD), a 5 radiation length preshower detector, a fine-grain set of scintillators at the back that can trigger on di-muon events, and two beam hole calorimeters, one made of $\mathrm{BaF}_{2}$ and one made of $\mathrm{PbF}_{2}$.

Each TRD consists of a felt plane, used as the radiator, followed by a xenon wire chamber to detect the transition photons. It is expected that the TRD system will improve our pion-electron identification by a factor of 100. The preshower detector consists of 10 planes of $2 \mathrm{~mm}$ scintillating fibers, alternating in orientation to provide fine-grain positional resolution, with each plane seperated by .25 radiation lengths of lead. This detector is expected to provide identifiable photons whose seperation is less than $1 \mathrm{~cm}$. The $\mathrm{PbF}_{2}$ beam hole calorimeter is the first implementation of this substance for use in high-energy calorimetry. It is the most compact crystal known for this use and is much more radiation resistant than lead glass. The performance comparison between the beam hole calorimeters will influence our choice for future experiments.

\section{FUTURE NEUTRAL KAON EXPERIMENTS AT}

\section{FERMILAB}

\subsection{Phase II of E799}

In the next fixed target run at Fermilab, currently scheduled for 1994, we again plan on performing two experiments. The first will be the continuation of E799, termed "Phase II". The values for expected numbers of events are approximately 20 times those in Table 2 .

The experiment will be located in a new beamline, able to withstand higher luminosities. In addition, the calorimeter will no longer be the lead glass array we currently are using, but will be composed of $\mathrm{CsI}$, a radiation hard scintillator that will achieve much better resolution (on the order of $1 \% / \sqrt{(E)}$ ). Besides this new calorimeter, an entirely new and enhanced set of photon vetoes will be installed.

\subsection{Proposed Experiment P832}

The second experiment scheduled for 1994 is a renewed search for direct $C P$ violation, using the same technique as for experiment E731. It will have two $K_{L}$ beams, one striking a regenerator. The improved resolution in measuring the photon energies in the $2 \pi^{\circ}$ decay means that a tighter mass cut can be made, reducing the systematic error due to background from merged $3 \pi^{\circ}$ decays. The improved photon veto system will help in this respect also. The goal for this experiment is a combined systematic and statistical error on the measurement of $\epsilon^{\prime} / \epsilon$ of about $10^{-4}$.

\subsection{KAMI (Kaons At the Main Injector)}

The most ambitious part of the kaon program at Fermilab will take advantage of the nearly continuous delivery of $120 \mathrm{GeV}$ protons available from the Main Injector, to be built at Fermilab later this decade. The siting of the beamline for the 1994 run takes into account the fact that it will be part of the Main Injector program. A new 
round of $\epsilon^{\prime} / \epsilon$ measurements are planned to achieve a precision of $2 \cdot 10^{-5}$ per year of running, while rare decay measurements will bring the single event sensitivity to a level of about $4 \cdot 10^{-14}$. It is possible that a $K_{S}$ experiment will be performed at this facility and a search for the decay $K_{L} \rightarrow \pi^{\circ} \nu \bar{\nu}$ is forseen.

\section{CONCLUSION}

In conclusion, a long-term program of neutral kaon rare decay and CP-violation experiments is planned at Fermilab, possibly extending into the next decade. We expect this program to deliver important physics results by building on the solid foundation laid by the current set of experiments and by E731.

\section{REFERENCES}

1. J.R. Patterson et al., Phys. Rev. Lett. 64 (1990) 1491
2. M. Woods et al., Phys. Rev. Lett. 80 (1988) 1695 see also the J.R. Patterson thesis, University of Chicago, (1990)

3. G. Costa and P.K. Kabir, Nuovo Cimento A 51 (1967) 564

4. H. Taureg et al., Phys. Lett. B 65 (1976) 92

5. A. Carroll et al., Phys. Rev. Lett. 44 (1980) 529

6. Y.C.R. Lin and G. Valencia, Phys. Rev. D 37 (1988) 143

7. C. Picciotto, Laboratoire National Saturne Preprint, LNS/Ph/91-15U (1991)

8. P. Ko and T.N. Truong, Phys. Rev. D 43 (1991) $\mathrm{R} 4$

9. Review of Particle Properties; Particle Data Group (1990) 